\title{
Recent advances in analytical methods for mycotoxins
}

\author{
Chris M. MARAgos \\ Mycotoxin Research Unit, USDA-ARS-NCAUR \\ (1815 N. University St., Peoria, IL, 61604, U.S.A.)
}

\begin{abstract}
Summary
Accurate and sensitive methods for detection of the major mycotoxins have been developed and improved for many years. Immunoassays, first introduced for mycotoxins in the 1970's, have found widespread use as screening tools. Despite the success of the enzyme-linked immunoassay (ELISA) format, new formats for immunoassays continue to be explored with the goal of improving upon the speed and ease-of-use of the assays. Recent advances in analytical technologies have allowed the adaptation of immunoassays to a wide variety of new formats. This review focuses on the recent developments in detection technologies for mycotoxins, in particular those using antibodies as the recognition element (immunosensors). Recent developments in surface plasmon resonance, fiber optic sensors, membrane-based immunoassays, fluorescence-based assays, and capillary-based immunoassays, are presented. The challenge remaining for many of these new technologies is one of making the transition from laboratory-based assays to validated field-portable screening assays.
\end{abstract}

Key words : review, biosensor, immunoassay, rapid tests

\section{Introduction}

Technologies for detection of mycotoxins developed rapidly when the associations were first made between these agents, produced by fungi, and disease in animals. Since that time analytical technologies have steadily advanced and the new detection technologies have been rapidly incorporated into mycotoxin testing strategies. Detection of mycotoxins is necessary for many reasons. Testing is done to help protect human and animal health, to facilitate greater agricultural productivity, and to meet contract specifications for minimum acceptable levels in foods and feeds. For convenience analytical methods can be subdivided into tests that can be performed rapidly by relatively untrained personnel (screening assays) and tests that require either more skill or more sophisticated instrumentation to perform (laboratory assays). Historically the screening assays have evolved from assays that were initially laboratory-based. 
Widely used screening assays include enzyme-linked immunosorbent assays (ELISAs), rapid cleanup columns combined with fluorescence detection, and thin layer chromatography. Widely used laboratory methods are predominantly chromatographic methods, including high performance liquid chromatography (HPLC) and gas chromatography. Increasingly HPLC with mass spectrometric detection is being used. Despite the considerable advantages of the chromatographic techniques, most remain laboratory-based assays because they require specialized training and/or expensive instrumentation to operate. Immunoassays, which rely upon the specificity of antibodies to bind target analytes, remain very popular because of the ease with which they can be performed. However, the low molecular weight of most mycotoxins (less than 1,000 daltons) somewhat restricts the immunoassay formats that can be used for their detection. This restriction can also be a factor in the development of biosensors based upon antibodies (immunosensors).

A large number of antibody-based devices have been tested recently for detection of

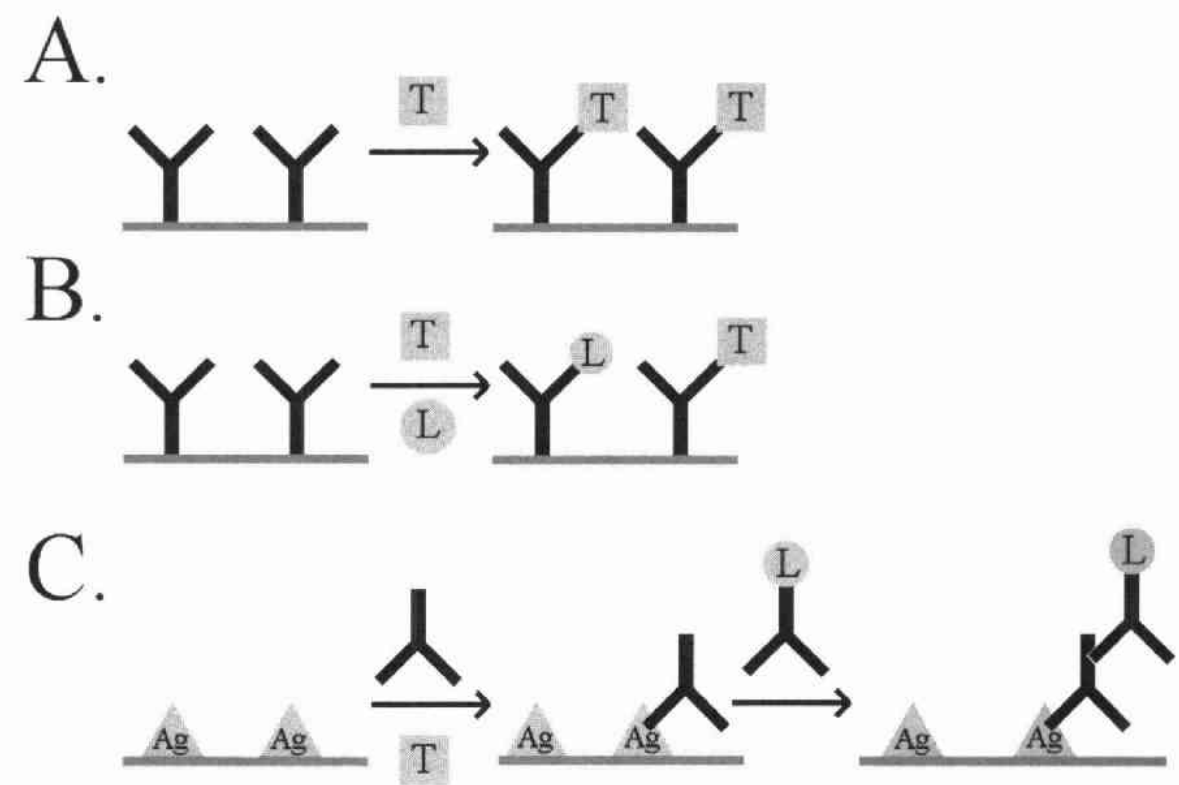

Fig.1. Three representative formats for small molecule immunoassay.

$\mathrm{T}, \mathrm{L}$, and $\mathrm{Ag}$ represent toxin, label, and toxin-antigen conjugate respectively. For brevity of presentation the washing steps, to remove non-specifically attached reagents, are not shown. (A) Non-competitive assay with immobilized antibody. This format does not provide signal amplification but can be adapted to concentrate toxin from a sample. (B) Competitive assay with immobilized antibody. This format is used in many commercial test kits and biosensors. (C) Competitive assay with immobilized antigen. As shown this format uses a secondary antibody, rather than the toxin-specific (primary) antibody with a label. Use of a labeled primary antibody simplifies the assay by reducing the number of steps. 
mycotoxins. The technologies that have been tested include evanescent wave-based devices such as fiber-optic immunosensors and surface plasmon resonance sensors. Portable sensors that use antibody or antigen-coated microbeads in miniature versions of affinity columns have also been developed as have similar technologies using liposomes containing fluorophores (flow injection liposome immunoassays). Other technologies using antibodies have been affinity-based capillary electrophoresis, and fluorescence polarization. This review will summarize the progress that has been made with each of these formats, highlighting those technologies that appear to have the potential to make the transition from laboratory-based assays to screening assays.

The earliest biosensors for mycotoxins were simple adaptations of enzyme-linked immunosorbent assay (ELISA) or immunoaffinity column (IAC) technologies. A commonly used IAC format involves antibody attached to a solid support. This format can also be used for non-competitive immunoassay (Fig. 1A). The ELISAs for low molecular weight materials are competitive assays where either the antibody (Fig. 1B) or the antigen (Fig. 1C) is immobilized. Biosensors also use these formats, often using labels or mechanisms other than enzymatic reactions as a means amplifying and detecting the binding event.

\section{Surface plasmon resonance and fiber optic probes}

Several biosensor formats use a phenomenon known as evanescence. The phenomenon occurs at the interface between two materials of differing refractive index. If light is applied to a surface at a certain angle of incidence it can be reflected, rather than refracted by the material. Under certain conditions the light can be totally internally reflected within a material, in this way light can be transmitted for substantial distances through optical fibers. The intensity of the evanescent wave diminishes dramatically with increasing distance from the surface of the fiber. Fiber optic and surface plasmon resonance (SPR) biosensors take advantage of the evanescent wave in different ways. Optical fibers can be coated with antibodies and the binding of antigen at the surface detected. For aflatoxins this was performed by applying excitation light at $360 \mathrm{~nm}$ to the fiber. Upon binding of aflatoxin to the sensor surface the fluorescence emission at 440 $\mathrm{nm}$ was detected, analogous to Fig. 1A. For fumonisins a competitive assay was used wherein $\mathrm{FB}_{1}$ in solution competed with a fluorescently tagged fumonisin $\left(\mathrm{FB}_{1}-\mathrm{FL}\right)$ for binding to the fiber, analogous to Fig. 1B. Both assays were shown to detect the indicated mycotoxins, however sensitivities were generally poor and problems attributed to refractive index-related effects were observed".

A more promising approach has been the use of the evanescent wave phenomenon in the SPR format. SPR assays measure changes in the refractive index at the interface between the sensor "chip" and the surrounding solution. Binding events near the 
interface alter the refractive index and are detected. Because of this, binding of large molecules to the surface is more efficiently detected than the binding of small molecules. Beginning in the 1990's several laboratories developed SPR assays for mycotoxins ${ }^{2-8)}$. With one exception ${ }^{3)}$ all used a format where antigen was coated onto the surface of the sensor chip and the binding of anti-mycotoxin antibody to the antigen was measured. The immobilized antigen technique was preferred because of the small molecular weight of the mycotoxins relative to the molecular weight of the antibody. That is, allowing the binding of the higher molecular weight antibody, rather than the lower molecular weight mycotoxin to be detected.

SPR assays have been developed for the aflatoxins, deoxynivalenol (DON), zearalenone (ZEN), ochratoxin $A(\mathrm{OA})$, and the fumonisins. Aflatoxin $\mathrm{B}_{1}\left(\mathrm{AFB}_{1}\right)$ detection through the binding of two anti-aflatoxin polyclonal antibodies yielded an assay with a linear range between 3 and $98 \mathrm{ng} / \mathrm{ml}^{4)}$. The same group also used SPR to develop single-chain fragment variable antibody domain ( $\mathrm{scFv}$ ) antibodies with affinity for $\mathrm{AFB}_{1}{ }^{9}$. The $\mathrm{scFv}$ were then used in an assay for the detection of AFB, in spiked extracts of grain, over the range of 0.75 to $48 \mathrm{ng} / \mathrm{ml}$. The presence of $5 \%(\mathrm{v} / \mathrm{v})$ methanol improved the response characteristics of the assay relative to buffer. Isolation of $\mathrm{scFv}$ with affinities for $\mathrm{AFB}_{1}$ was also performed by Moghaddam et al. ${ }^{5)}$ by panning phagedisplay libraries for binding to $\mathrm{AFB}_{1}-\mathrm{BSA}$. The scFv isolated were capable of detecting a $50 \mathrm{nM}$ solution of $\mathrm{AFB}_{1}$ or $\mathrm{AFG}_{1}$.

Several SPR assays have been reported for DON. All used a format with antigen attached to the surface of the chip. The antigens attached were DON-biotin ${ }^{6}$, DONcasein $^{8)}$, or DON-BSA ${ }^{7}$. The first of these was tested with wheat samples prepared using solid phase extraction (SPE) columns (Romer MycoSep), and yielded an assay with a working range of 0.13 to $10 \mu \mathrm{g} / \mathrm{ml}$ (midpoint $0.72 \mu \mathrm{g} / \mathrm{ml}$ ) ${ }^{6}$. MycoSep columns were also used by van der Gaag et al. " to purify wheat extracts before SPR assay. The cleanup may not be necessary in some cases. Tudos et al. ${ }^{8)}$ recently reported an SPR assay where wheat was extracted with acetonitrile/water, and the extract simply diluted before assay. Dilution was necessary to minimize the effects of solvent upon the affinity of the antibodies for DON. The latter assay had a working range of 2.5 to $30 \mathrm{ng} \mathrm{DON} / \mathrm{ml}$ and correlated well with HPLC-MS when tested with a limited number of wheat samples.

The only published report using a non-competitive SPR assay for mycotoxins (ie similar to Fig. 1A) is that of Mullett et al. . $^{3}$. Anti-fumonisin antibodies were immobilized on the surface of the chip, and binding of $\mathrm{FB}_{1}$ was measured directly. This may be possible because of the higher molecular weight of the fumonisins ( 721 daltons for $\mathrm{FB}_{1}$ ). The assay was able to detect $\mathrm{FB}_{1}$ in standard solutions at $50 \mathrm{ng} / \mathrm{ml}$. The assay was rapid, with an analysis time of $10 \mathrm{~min}$. Fumonisins were also tested as part of a multimycotoxin assay developed by van der Gaag et al. "). In the latter report four mycotoxins were detected simultaneously using a sensor containing four serially connected flow 
cells. The chip had toxin or toxin-protein conjugates immobilized, and binding of antibody was detected. The four antibodies were mixed with the sample before injection.

\section{Test strips}

Recently several laboratories have developed membrane-based test strips for mycotoxin detection. The membrane assays can be grouped into those formats where the solutions flow through the membrane (enzyme linked immunofiltration assays or ELIFA), those where the test strips are incubated in tubes containing the reagents (dipstick tests), and those where the solutions flow parallel to the membrane (lateral flow devices). Some of the ELIFA tests have been available commercially for several years, for example for detection of aflatoxin $\mathrm{M}_{1}\left(\mathrm{AFM}_{1}\right)$ in milk (Aflacup, International Diagnostic Systems Corp., St. Joseph, MI). ELIFA assays for OA and T-2 toxin have also been collaboratively studied ${ }^{10}$.

Dipstick assays, where the antibodies are attached to the test strip and the reagents are exposed to the device in a tube, have been developed for fumonisin $B_{1}\left(F B_{1}\right)$ and for $\mathrm{T}-2$ toxin ${ }^{11,12)}$. The technique has also been used for detection of multiple toxins, such as $\mathrm{AFB}_{1}, \mathrm{~T}-2$ toxin, 3-acetyl-DON, roridin A, and zearalenone ${ }^{13)}$.

With lateral flow devices the sample extract is applied at one end of the device and the solution migrates to the other end of the device by capillary action. This type of format is favored, particularly if further addition of reagents is not necessary. A lateral flow immunoassay for $\mathrm{AFB}_{1}$ using aflatoxin-modified liposomes was recently reported that could detect as little as $20 \mathrm{ng} \mathrm{AFB}_{1}$ applied to the device ${ }^{14)}$. Several manufacturers have recently made lateral flow test kits available commercially. R-Biopharm (Darmstadt, Germany) has developed lateral flow devices for DON, fumonisins, and aflatoxins. Charm Science, Inc. (Lawrence, MA) has developed a lateral flow device for measuring $\mathrm{AFM}_{1}$ in milk. The Charm Science product uses a small incubator to achieve the required reaction temperature $\left(45^{\circ} \mathrm{C}\right)$, and a portable instrument to measure the color development of the test strip. Milk is added to the test strip without any cleanup or dilution. By comparing the color development at a control line to color development at a test line the kit can give a positive/negative response at $0.5 \mathrm{ng} \mathrm{AFM} / \mathrm{ml}$. The ease of use of the lateral flow test strips will undoubtedly make them valuable for screening commodities and foods.

\section{Fluorescence-based immunoassays}

Two types of fluorescence-based immunoassay that have recently been used for mycotoxin detection are the fluorescence polarization (FP) immunoassays and timeresolved fluoroimmunoassays (TR-FIA). Each uses a different property of fluorescence. 
With fluorescence polarization the fluorescence intensity is measured in the vertical and horizontal planes, after exposing the fluorophore to polarized light at the excitation wavelength. The rate of rotation of fluorophore in solution directly affects the degree of polarization of the fluorescence emission. Therefore FP instruments are indirectly measuring the rate of rotation of the fluorophore ("tracer"). If the tracer is a mycotoxinfluorophore conjugate, the binding of antibody to the mycotoxin can slow the rate of rotation of the fluorophore and increase the polarization. Competitive FP immunoassay is based upon the competition of tracer and toxin (from the sample) for antibody in solution analogous to Fig. 1B. A major difference is that successful FP does not require immobilization of the antibody or any of the other reagents. Because of the principle of the assay, physical separation of the antibody-bound tracer and the free tracer is not necessary. The latter property can make the FP immunoassays very rapid. FP immunoassays have recently been described for the fumonisins, DON, and the aflatoxins $^{15-17}$.

The TR-FIAs rely upon a different property of fluorescence, namely the rate of decay of a fluorophore after a pulse of light at the excitation wavelength. Certain fluorophores, such as europium, have relatively long fluorescence lifetimes, allowing their fluorescence to be discriminated from that of the more rapidly decaying fluorescence of potential interferences. TR-FIA can essentially operate the same as competitive ELISA. TR-FIAs have been developed for $\mathrm{AFB}_{1}$ in soya seeds, dried figs and raisins ${ }^{18)}$, and for $\alpha$-zeralanol (zeranol) and $\alpha$-zearalenol in bovine urine ${ }^{19}$.

\section{Capillary-based immunoassays}

Affinity columns have generally been prepared in one of two formats: with either the mycotoxin antibody attached to the column material (Fig. 1A) or with a toxin or toxin-protein conjugate attached to the column material (Fig. 1C). Biosensors using both formats have been described. The first format, with antibody immobilized on the beads, is essentially that of commercially available IAC $^{20}$. A handheld biosensor using this format was recently described for measurement of aflatoxins ${ }^{21}$. Aflatoxin solutions were passed over the column and then eluted from it with methanol/water. The fluorescence of the eluate was then determined by a built-in detector. Essentially this design combined all of the steps of an IAC cleanup and determination in a single device. The advantage of this design is that the assay is non-competitive and the aflatoxin was determined directly.

Several biosensors have also been described using the indirect format: with antigen, rather than antibody, immobilized onto the microbeads (Fig. 1C). An advantage of this format is that it is more amenable to re-use, because fresh antibody is used with each sample. However, the response is inversely proportional to the amount of toxin present. A commercial instrument manufactured by Sapidyne Instruments (Idaho City, ID, USA) 
was the basis for assays developed for detection of aflatoxin $B_{1}$ in nut puree, peanut butter, and pistachio meal ${ }^{22}$. The same instrument was also used to develop an assay for ZEN standards ${ }^{23}$. A similar format, using an enzymatic rather than a fluorescent label has also been applied to detection of $\mathrm{AFB}_{1}$ in pistachios ${ }^{24}$. A 15 min extraction procedure was combined with a $10 \mathrm{~min}$ assay, and was used over the range of 4 to 400 ng $\mathrm{AFB}_{1} / \mathrm{g}$ sample. While enzymatic labels have the advantage of providing signal amplification they have the disadvantage of requiring an additional incubation step while the product forms.

Another type of flow injection assay uses antigen-tagged liposomes rather than microbeads for analysis of fumonisins in corn ${ }^{25}$. Antibodies were coated onto the surface of capillaries and sequential injections were made of fumonisin-contaminated samples and fumonisin-tagged liposomes filled with sulforhodamine B. After a washing step and lysis of the liposomes the fluorescence of the sulforhodamine $B$ was determined. Recovery of $\mathrm{FB}_{1}$ over the range of 1 to $4 \mu \mathrm{g} / \mathrm{g}$ cornmeal ranged from 80 to $92 \%$. The assays were performed within $11 \mathrm{~min}$, and the limit of detection was $0.1 \mathrm{ng}$, suggesting this may be a sensitive and rapid method for estimating fumonisins in cornbased foods.

Capillary-based assays can also be conducted using the instrumentation of capillary electrophoresis (CE). Combining immunoassay with CE can integrate the selectivity of antibodies with the separation power of CE. Extracts of maize were combined with fumonisin antibodies and $\mathrm{FB}_{1}$-fluorescein conjugate $\left(\mathrm{FB}_{1}-\mathrm{FL}\right)$ and the mixture injected onto a $\mathrm{CE}^{26)}$. The $\mathrm{CE}$ separated the unbound $\mathrm{FB}_{1}-\mathrm{FL}$ and the bound $\mathrm{FB}_{1}-\mathrm{FL}$, which were then detected as separate fluorescent peaks. With increasing $\mathrm{FB}_{1}$ content, the unbound $\mathrm{FB}_{1}-\mathrm{FL}$ increased and the bound $\mathrm{FB}_{1}-\mathrm{FL}$ decreased, allowing estimation of the fumonisin content. The assays were relatively rapid $(6 \mathrm{~min})$, unfortunately the sensitivity was insufficient for all but very highly contaminated samples. Such a format may benefit from the use of higher affinity fumonisin antibodies ${ }^{26}$. The CE format is particularly interesting because of the possibilities of incorporating ongoing improvements in microfluidics and miniaturization to perform multi-analyte detection ${ }^{27}$.

\section{Future challenges}

The development of immunoassays has continued to progress as instrumentation has continued to evolve. Several of the formats described in the previous sections have the potential to improve upon the commonly used immunoassay formats. It may also be possible in the future to replace the biological element of the biosensor (eg the antibody) with man-made molecular recognition elements, for example molecularly imprinted polymers ${ }^{28,29)}$. The challenge for all of these technologies is to bridge the gap between limited-use laboratory-based assays and widely used screening assays. The technologies 
that are the easiest to use, the fastest, and the least expensive can be expected to be the most successful. A common hurdle facing new technologies is the process of validation. Several, but not all, of the described technologies may withstand the rigors of validation required to become commercially viable.

\section{References}

1 ) Maragos, C.M., Thompson, V.S.: Natural Toxins, 7, 371-376 (1999)

2) Van der Gaag, B., Burggaaf, R.A., Wahlstrom, L.: Poster Presentation at the 110th AOAC International Annual Meeting and Exposition, Orlando, Fl. Sept. 8-12 (1996)

3 ) Mullett, W., Lai, E.P.C., Yeung, J.M.: Anal. Biochem., 258, 161-167 (1998)

4 ) Daly, S.J., Keating, G.J., Dillon, P.P., Manning, B.M., O’Kennedy, R.O., Lee, H.A., Morgan, M.R.A.: J. Agric. Food Chem., 48, 5097-5104 (2000)

5 ) Moghaddam, A., Lobersli, I., Gebhardt, K., Braunagel, M., Marvik, O.J. J. Immunol. Meth., 254, 169-181 (2001)

6 ) Schnerr, H., Vogel, R.F., Niessen, L.: Food Agric. Immunol., 14, 313-321 (2002)

7 ) Van der Gaag, B., Spath, S., Dietrich, H., Stigter, E., Boonzaaijer, G., van Osenbruggen, T., Koopal, K.: Food Control, 14, 251-254 (2003)

8 ) Tudos, A.J. Lucas-van den Bos, H.R., Stigter, E.C.A.: J. Agric. Food Chem., 51, 5843-5848 (2003)

9 ) Daly, S.J., Dillon, P.P., Manning, B.M., Dunne, L., Killard, A., O’Kennedy, R.O.: Food Agric. Immunol., 14, 255-274 (2002)

10) De Saeger, S., Sibanda, L., Desmet, A., Van Peteghem, C.: Intl. J. Food Microbiol., 75, 135-142 (2002)

11) Schneider, E., Usleber, E., Martbauer, E. J.: Agric. Food Chem., 43, 2548-2552 (1995)

12) De Saeger, S., Van Peteghem, C.: Appl. Environ. Microbiol., 62, 1880-1884 (1996)

13) Schneider, E., Usleber, E., Martlbauer, E., Dietrich, R., Terplan, G: Food Addit. Contam., 12, 387-393 (1995)

14) Ho, J.A.A., Wauchope, R.D.: Anal. Chem., 74, 1493-1496 (2002)

15) Maragos, C.M., Jolley, M.E., Plattner, R.D., Nasir, M.S.: J. Agric. Food Chem., 49, 596-602. (2001)

16) Maragos, C.M., Plattner, R.D.: J. Agric. Food Chem., 50, 1827-1832 (2002)

17) Nasir, M.S., Jolley, M.E.: Combinatorial Chemistry \& High Throughput Screening, 6, 267-273 (2003)

18) Bacigalupo, M.A., Ius, A., Meroni, G., Dovis, M., Petruzzelli, E.: Analyst, 119, 2813-2815 (1994)

19) Cooper, K.M., Tuomola, M., Lahdenpera, S., Lovgren, T., Elliott, C.T., Kennedy, D.G.: Food Add. Contam., 19, 1130-1137 (2002)

20) Scott, P.M., Trucksess, M.W.: J. AOAC Intl., 80, 941 -949 (1997)

21) Carlson, M.A., Bargeron, C.B., Benson, R.C., Fraser, A.B., Phillips, T.E., Velky, J.T., Groopman, J.D., Strickland, P.T., Ko, H.W.: Biosen. Bioelect., 14, 841-848 (2000)

22) Strachan, N.J.C., John, P.G., Miller, I.G.: Food Agric. Immunol., 9, 177-184 (1997)

23) Carter, R.M., Blake II, R.C., Mayer, H.P., Echevarria, A.A., Nguyen, T.D., Bostanian, L.A.: Anal. Lett., 33, 405-412 (2000) 
24) Garden, S.R., Strachan, N.J.C.: Analytica Chimica Acta, 444, 187-191 (2001)

25) Ho, J.A., Durst, R.A.: Anal. Biochem., 312, 7-13 (2003)

26) Maragos C.M.: Food Agric. Immunol., 9, 147-157 (1997)

27) Narang, U., Gauger, P.R., Kusterbeck, A.W., Ligler, F.S.: Anal. Biochem., 255, 13-19 (1998)

28) Jodlbauer, J., Maier, N.M., Lindner, W.: J. Chromatogr. A., 945, 45-63 (2002)

29) Weiss, R., Freudenschuss, M., Krska, R., and Mizaikoff, B.: Food Addit. Contam., 20,386-395 (2003) 